\title{
Intertemporal and Spatial Location of Disposal Facilities
}

\author{
Francisco J. André, Francisco Velasco and Luis Gonzalez-Abril \\ Department of Economics. Universidad Pablo de Olavide. Carretera de Utrera, km. 1. 41008 Sevilla (Spain). \\ E-mail: andre@upo.es \\ Department of Applied Economics I. University of Sevilla. Avda. Ramón y Cajal 1, 41018 Sevilla (Spain). E-mail: \\ $\{$ velasco,luisgon\}@us.es
}

The date of receipt and acceptance will be inserted by the editor

\begin{abstract}
The optimal capacities and locations of a sequence of landfills are studied, and the interactions between these characteristics are considered. Deciding the capacity of a landfill has some spatial implications since it affects the feasible region for the remaining landfills, and some temporal implications because the capacity determines the lifetime of the landfill and hence the moment of time when the next landfills should be constructed. Some general mathematical properties of the solution are provided and interpreted from an economic point of view. The resulting problem turns out to be non-convex and, therefore, it cannot be solved by conventional optimization techniques. Some global optimization methods are used to solve the problem in a particular case in order to illustrate how the solution depends on the parameter values.
\end{abstract}

Keywords: Landfilling, Exhaustible Resources, Optimal Capacity, Optimal Location, Global Optimization.

JEL codes: C61, C63, Q30, R53

\section{Introduction}

Waste management is a growing environmental concern as well as an issue of primary importance from social, technical and economic points of view (see, for example, Beede and Bloom 1995, Porter 2002, Fullerton and Kinnaman 2002, André and Cerdá 2006). From among all the available techniques for solid waste treatment, landfilling ${ }^{1}$ has traditionally been the most widely used. It therefore follows that the characteristics of treatment or disposal facilities are fundamental decisions to be taken in waste management (see Swallow et al., 1992; Highfill et al., 1994; Kunreuther and Easterling, 1996; Quah, 2002).

The operation of disposal facilities in relation with their location and capacity, has changed dramatically over the last 20 years for economic and environmental reasons. The location of landfills has tended to move further away from cities due to the scarcity of land in densely populated urban areas and the 
increased concern about the effects on our health and the environment. Regarding capacity, small landfills have closed and large landfills have grown in number and size. At the start of the 1970s, there were 20,000 landfills in the United States, but by the end of the 1980s only 6,000 and by 1998 barely 2,000. By the end of the 1980s, a few hundred landfills handled half of all the municipal solid waste generated in the United States (see US EPA, 1988; Repa, 2000; Porter, 2002). This paper studies the joint problem of determining the location and capacity of landfills and analyzes the specific economic issues raised by this problem.

In economics the capacity of a landfill can be rationalized as an exhaustible resource. Nevertheless, it turns out to be a rather special kind of resource which requires specific analysis and gives rise to specific economic features. The first peculiarity is that, unlike other natural resources, landfills do not typically have an existence value, since people do not enjoy nor receive any direct utility from a landfill itself. Rather, landfills play a role as disposal facilities for the elimination of waste. The economic value of a landfill should therefore be assessed by its ability to eliminate waste, and not by its mere existence. Secondly, unlike other resources whose initial stock and location is given by nature, the capacity and the location of a landfill can be chosen by decision-makers. Finally, although landfills are depletable, they are replaceable as well (see Ready and Ready, 1995). Unlike other resources whose depletion is irreversible, once a landfill is full it can be replaced at some cost by a new one which will carry out exactly the same function. The capacity of the new landfill will also be depleted and so on.

Deciding the capacity of a landfill has some relevance in the setup costs and also in the lifetime of landfills. On the one hand, the smaller the capacity of the landfill to be constructed, the smaller the construction cost. However, on the other hand, the lifetime of such a landfill would be short, and hence the construction of a new landfill would have to be undertaken sooner. This conflict between present and future costs gives rise to a sequential (dynamic) decision problem implying that a planning time horizon has to be divided into several subintervals, the length of which is endogenously determined. The sequential nature of landfill use is recognized in Ready and Ready (1995), Huhtala (1997), Gaudet et al. (1998, 2001) and André and Cerdá (2001, 2004), however in all these papers, except André and Cerdá (2001, 2004), landfill capacity is a given and therefore the capacity decision is not explicitly considered.

In André and Cerdá $(2001,2004)$ the optimal capacity of landfills is studied in a sequential way, but the location decision is not considered. The present paper builds on the research by André and Cerdá (2001, 2004) and presents a more general model enriched by including continuous location decisions. This results in an intertemporal and spatial problem where capacity and location decisions for landfills are jointly made in a sequential way and we show how these decisions interact with each other. The main idea is that the capacity decision has some spatial and some temporal implications: spatial, because the larger the landfill, the smaller the remaining feasible region to locate further landfills; and temporal, because the capacity determines the lifetime and hence the time when the next landfills will be needed.

Facility location has been thoroughly studied in economics and operations research (see Drezner, 2002 or Revelle and Eiselt, 2005 for a survey). Our problem is similar to the so-called Dynamic Capacitated Plant Location Problem (DCPLP) which consists of optimally locating a number of capacitated facilities to supply several consumers, in such a way that the sum of the fixed costs and operating costs is minimized (see, for example, Fong and Srinivasan, 1986, Shulman, 1991 or Lim and Kim 1999). Nevertheless, our problem departs from the common framework of DCPLP in several aspects: firstly, in these models, facilities are usually considered as non-replaceable whereas depletion and replacement of landfills is crucial in our approach, in the sense that a full landfill can always be replaced by a new one. Secondly, typical 
DCPL problems are formulated in discrete time whereas we model time as a continuous variable so that the optimal lifetime of landfills can be determined in a more flexible and explicit way. Thirdly, we account for the amount of land occupied by each landfill, including the landfill surface itself and the surrounding area, in which no other landfill should be placed for security reasons. To model this fact a dispersion constraint is included, which makes the problem non-convex. Moreover, in the literature, the aim is typically to propose operational methods to solve the computational problem ${ }^{2}$, whereas we focus on the general properties of the solution and the economic interpretation of these properties.

Since our model can be seen as one of multiple facilities competing for limited space or land (in an intertemporal setting), it makes sense to look for a measure of the cost associated to land scarcity. Moreover, this scarcity should be allowed to vary across different regions. The first notable result of our model is the ability to provide an explicit measure of the scarcity cost or shadow price of land around each facility, which depends on the specific time and the place where it is located.

Second, we provide a generalization of the Optimal Capacity Condition by André and Cerdá (2001, 2004), which rules the optimal sequence of capacities when the location decision is taken into account. This condition can also be rewritten as a difference equation for the scarcity cost of land across the sequence of landfills. This shadow price is typically decreasing across landfills since, with a positive discount rate, the first landfills tend to be located in the most preferred places.

Third, it is shown that the structure of the problem allows for the possibility of an optimal excess capacity with respect to the disposal requirements. This may happen if the discounted cost reduction obtained when increasing the capacity of any landfill overcompensates the increase of construction cost and this results in an excess capacity which cannot be eliminated by reducing the capacity of the last landfill(s) of the sequence, since it is (or they are) at the lower bound.

Concerning the optimal order of landfills, the model is consistent with the fact that, as time goes on, landfills are, in general, constructed further away from cities, which turns out to be a consequence of the classic result by Herfindahl (1967) for a sequence of resource deposits. When there are several cities, we cannot identify a single distance, and the equivalent result in this framework means that the weighted distance, as measured by the instantaneous transportation cost of waste, is increasing. This is typically the case in our framework. Nevertheless, it is also shown that, due the presence of setup costs, the fulfillment of this condition is not guaranteed unless the optimal sequence of capacities is constant.

In Section 2 the mathematical structure of the problem is presented and shown to be non-convex in nature, and therefore conventional optimization techniques are not suitable. In Section 3 the basic mathematical and economic properties of the solution and their interpretation are analyzed. In Section 4 an illustrative example is solved by using global optimization techniques. Some sensitivity analysis exercises are performed to gain further insights into the effect of different parameters. A larger fixed construction cost makes it optimal to reduce the number of landfills and makes the sequence of capacities decrease more, and the opposite happens for the marginal construction and transportation costs. The observed trends for the number of landfills to decrease and landfill capacities to increase can be understood as a consequence of a sudden increase in setup cost due to the scarcity of land and tougher environmental and technical regulations. An enlargement of the planning horizon causes a stair-shaped increment for the number of landfills and a sawtooth shape for the average capacity. Section 5 summarizes the conclusions. 


\section{The problem}

\subsection{Preliminaries}

A decision problem involving a sequence of landfills is modelled. For each landfill, two decisions need to be made: the capacity and the location. Concerning the location, two kinds of restrictions need to be satisfied: first, landfills should not be too close to any urban area or other forbidden region (facilities, natural resources, etc.) and second, a landfill cannot be placed exactly in the same place (or extremely close to) where another landfill has been previously located due to safety reasons. For example, Vasiloglou (2004) includes as a location criterion for landfills "the distance from unstable areas and areas with subsidence zones or expansive soils" (p. 433). The area around a used landfill can be considered as unstable because of methane emissions. Note, however, that there is a fundamental difference between these two location constraints: the former is exogenously given, since the cities and facilities are assumed to be previously located when the landfill-siting decision is made, whereas the latter is an endogenous constraint because deciding the location of any landfill influences the location possibilities for the remaining landfills.

In this formulation the possibility of having several landfills operating at the same time is disregarded. From a technical point of view, this makes the problem simpler and allows us to focus on the sequential nature of the problem. Concerning the realism of this assumption, the problem can be interpreted as that of such a small region that it is not reasonable or operational to operate more than one landfill at a time. The case for several simultaneously open landfills is left for future work (see Section 5).

\subsection{Mathematical formulation}

Assume there are $m$ cities located at different given points of the map $P_{j} \equiv\left(p_{j 1}, p_{j 2}\right), j=1, \ldots, m$. At time $t \in[0, \tau]$, city $j$ generates a flow of waste $q_{j}(t)$.

At time $t=0$ a landfill (denoted as landfill 0 ) is needed, with arbitrary capacity $Y_{0}$, located at an endogenously decided point $R_{0} \equiv\left(r_{01}, r_{02}\right) \in \Omega$, where $\Omega$ is an exogenously bounded feasible region (which accounts for the need to be far enough from any populated or protected area). Construction or setup costs depend on $Y_{0}$, in accordance with the increasing, convex and twice differentiable cost function $C\left(Y_{0}\right) .{ }^{3}$ The instantaneous transportation cost of waste to this landfill equals $\phi \sum_{j=1}^{m} q_{j}(t) d\left(P_{j}, R_{0}\right)$, where $d\left(P_{j}, R_{0}\right)$ is the Euclidean distance from $P_{j}$ (city $j$ ) to $R_{0}$ and $\phi$ is a parameter measuring transportation cost per unit of waste and distance.

The capacity of this landfill will become exhausted at some time. In principle, one could consider the specific instant when landfill 0 stops being used and a new one starts to be used instead as a discretionary decision. Nevertheless, as landfills do not usually have any existence value, the operational assumption can be made that the planner will use the landfill until its whole capacity is depleted and only then will the construction and use of a new landfill be initiated. In Section 3.3 it is argued that this is naturally the case for all landfills except, perhaps, the final landfill. Hence landfill 0 can be considered as fully depleted at time $T_{1}$, implicitly determined by the condition $\int_{0}^{T_{1}} Q(t) d t=Y_{0}$, where $Q(t) \equiv \sum_{j=1}^{m} q_{j}(t)$ is the total amount of waste generated at time $t$.

At $T_{1}$ a new landfill is needed. Denote as $Y_{1}$ and $R_{1} \equiv\left(r_{11}, r_{12}\right)$ the capacity and the location of this new landfill. The feasible region where this landfill can be located is now smaller than the original one, since the new landfill cannot be constructed in the same space occupied by the previous landfill or within a safety region around it. Furthermore, the larger the capacity, the larger the amount of occupied space 
and the wider the safety region needed due to potential risks. This limitation is modelled by imposing the constraint $d\left(R_{0}, R_{1}\right) \geq \beta\left(Y_{0}+Y_{1}\right)$, where $\beta$ is a parameter determining the size of the security area required around each landfill. The value of $\beta$ could vary across regions depending on environmental conditions (the direction of the wind, the presence of subterraneous water flows and so on, see Vasiloglou, 2004). The new landfill will last until time $T_{2}$, given by $\int_{T_{1}}^{T_{2}} Q(t) d t=Y_{1}$, and so on, up to the final landfill, denoted by $K-1$, where $K$ is a decision variable. In general, a landfill constructed at time $T_{i}$, located at $R_{i} \equiv\left(r_{i 1}, r_{i 2}\right)$, with capacity $Y_{i}$, will last until $T_{i+1}$, determined by $\int_{T_{i}}^{T_{i+1}} Q(t) d t=Y_{i}$. Construction and instantaneous transportation costs are given by $C\left(Y_{i}\right)$ and $\phi \sum_{j=1}^{m} q_{j}(t) d\left(P_{j}, R_{i}\right)$ respectively and $R_{i}$ has to satisfy $d\left(R_{i}, R_{k}\right) \geq \beta\left(Y_{i}+Y_{k}\right)$ for $k=1, \ldots, i-1$.

The planner's problem consists of finding a number of landfills $K$, a sequence of capacities $Y \equiv$ $\left\{Y_{0}, \ldots, Y_{K-1}\right\}$, switching times $T \equiv\left\{T_{1}, \ldots, T_{K}\right\}$ and locations $R \equiv\left\{R_{0}, \ldots, R_{K-1}\right\}$ to minimize total discounted cost in the time horizon $[0, \tau]$, i.e.,

$$
\min \sum_{i=0}^{K-1} e^{-\delta T_{i}}\left[C\left(Y_{i}\right)+\int_{T_{i}}^{T_{i+1}} e^{-\delta\left(t-T_{i}\right)}\left(\phi \sum_{j=1}^{m} q_{j}(t) d\left(P_{j}, R_{i}\right)\right) d t\right]
$$

subject to

$$
\begin{aligned}
\int_{T_{i}}^{T_{i+1}} Q(t) d t & =Y_{i}, \quad i=0,1,2, \ldots, K-1, \\
T_{0} & =0, T_{K} \geq \tau, \quad \underline{Y} \leq Y_{i} \leq \bar{Y}, \quad R_{i} \in \Omega, \\
d\left(R_{i}, R_{k}\right) & \geq \beta\left(Y_{i}+Y_{k}\right), \quad i, k=0, \ldots, K-1, i<k
\end{aligned}
$$

where $\delta$ is the discount rate, and $\underline{Y}$ and $\bar{Y}$ represent some minimum and maximum capacity constraints, which may be given by legal or technical reasons. The constraint $T_{K} \geq \tau$ means that the overall capacity of the whole sequence needs to be large enough to meet the requirements in the time horizon $[0, \tau]$.

To keep the analysis as simple as possible, $q_{j}(t)$ is assumed to be constant through time ${ }^{4}: q_{j}(t) \equiv q_{j}$ $\forall j, Q(t) \equiv Q$. Therefore, (2) can be written as $Q\left(T_{i+1}-T_{i}\right)=Y_{i}$ which, in turn, can be solved for $T_{i+1}$, which gives

$$
T_{i+1}=T_{i}+\frac{Y_{i}}{Q}, \quad i=0, \ldots, K-1 .
$$

Using (3) instead of (2), (1) can be seen as an optimal control problem, where $T_{i}$ is the state variable and $R_{i}, Y_{i}$ the control variables. Using (3) recursively we obtain $T_{1}=\frac{Y_{0}}{Q}, T_{2}=\frac{Y_{0}+Y_{1}}{Q}, \ldots$, and in general, $T_{i}=\sum_{l=0}^{i-1} \frac{Y_{l}}{Q}$, for $i=1, \ldots, K$. This expression can be substituted in (1) to eliminate $T_{i}$ and obtain the following expression for the objective function ${ }^{5}$ :

$$
J \equiv C\left(Y_{0}\right)+\frac{T C_{0}}{\delta}\left(1-e^{-\delta \frac{Y_{0}}{Q}}\right)+\sum_{i=1}^{K-1} e^{-\frac{\delta}{Q} \sum_{\ell=0}^{i-1} Y_{\ell}}\left[C\left(Y_{i}\right)+\frac{T C_{i}}{\delta}\left(1-e^{-\delta \frac{Y_{i}}{Q}}\right)\right]
$$

$T C_{i} \equiv \phi \sum_{j=1}^{m} q_{j} d\left(P_{j}, R_{i}\right)$ denoting transportation cost for landfill $i$. Problem (1) involves deciding the value of a discrete variable $(K)$ and some continuous variables $(R, Y)$. We solve this problem for all possible values of $K$, and choose that which provides the lowest cost. For every value of $K$ we have the problem

$$
\begin{aligned}
\mathcal{J}_{K} \equiv \min _{\{Y, R\}} & J \\
\text { s.t. } \quad & \sum_{i=0}^{K-1} Y_{i} \geq \tau Q, \\
& \underline{Y} \leq Y_{i} \leq \bar{Y}, \quad R_{i} \in \Omega, \\
& d\left(R_{i}, R_{k}\right) \geq \beta\left(Y_{i}+Y_{k}\right), \quad i<k
\end{aligned}
$$


$\mathcal{J}_{K}$ being the optimal value of the objective function with $K$ landfills. The last set of constraints represents the interaction between capacity and location, as illustrated in Figure 1. Consider a solution with two landfills located at $R_{0} \equiv\left(r_{01}, r_{02}\right)$ and $R_{1} \equiv\left(r_{11}, r_{12}\right)$, with capacities $Y_{0}$ and $Y_{1}$. The circles around $R_{0}$ and $R_{1}$ represent the safety regions. Suppose that $Y_{0}$ increases while $R_{0}$ and $Y_{1}$ remain unchanged. Hence $R_{1}$ becomes unfeasible as a location for the second landfill. The capacity and location of a landfill affect the feasible capacities and locations for the rest of the landfills. Figure 1 also illustrates the non-convex nature of the problem. Even if $\Omega$ is a convex set, once a landfill is located, the remaining feasible set $\left\{\Omega-B_{\beta Y_{0}}\left(R_{0}\right)\right\}$, where $B_{\beta Y_{i}}\left(R_{i}\right)$ denotes the ball centered at $R_{i}$ with radius $\beta Y_{i}$, is non-convex. If the condition $d\left(R_{i}, R_{k}\right) \geq \beta\left(Y_{i}+Y_{k}\right)$ is binding, we say that landfills $i$ and $k$ are 'as-close-as-possible'.

\section{Properties and economic interpretation}

\subsection{Optimality conditions}

For any value of $K$, we construct the Lagrangian

$$
\mathcal{L}=J+\mu\left(\tau Q-\sum_{i=0}^{K-1} Y_{i}\right)+\sum_{i=0}^{K-1} \alpha_{0 i}\left(\underline{Y}-Y_{i}\right)+\sum_{i=0}^{K-1} \alpha_{1 i}\left(Y_{i}-\bar{Y}\right)+\sum_{\substack{i, k=0 \\ i<k}}^{K-1} \lambda_{i k}\left[\beta\left(Y_{i}+Y_{k}\right)-D_{i k}\right]
$$

where $\mu, \alpha_{0 i}, \alpha_{1 i}$ and $\lambda_{i k}$ are the Lagrange multipliers and $D_{i k} \equiv d\left(R_{i}, R_{k}\right)$ is the distance between landfills $i$ and $k$. Given the non-convexity of the problem, several local minima may exist and a global minimum cannot be found just by solving first-order (Kuhn-Tucker) conditions. Nevertheless, these conditions are studied in order to obtain some insight into the properties of the solution:

$$
\begin{gathered}
C^{\prime}\left(Y_{0}\right)+e^{-\frac{\delta}{Q} Y_{0}} \frac{T C_{0}}{Q}+\beta \sum_{i=1}^{K-1} \lambda_{0 i}= \\
\frac{\delta}{Q} \sum_{i=1}^{K-1} e^{-\frac{\delta}{Q} \sum_{\ell=0}^{i-1} Y_{\ell}}\left[C\left(Y_{i}\right)+\left(1-e^{-\frac{\delta}{Q} Y_{i}}\right) \frac{T C_{i}}{\delta}\right]+\mu+\alpha_{00}-\alpha_{10} \\
e^{-\frac{\delta}{Q} \sum_{\ell=0}^{h-1} Y_{\ell}}\left(C^{\prime}\left(Y_{h}\right)+e^{-\frac{\delta}{Q} Y_{h}} \frac{T C_{h}}{Q}\right)-\frac{\delta}{Q} \sum_{i=h+1}^{K-1} e^{-\frac{\delta}{Q} \sum_{\ell=0}^{i-1} Y_{\ell}}\left[C\left(Y_{i}\right)+\left(1-e^{-\frac{\delta}{Q} Y_{i}}\right) \frac{T C_{i}}{\delta}\right] \\
h=1, \ldots K-1
\end{gathered}
$$

where $d_{j h} \equiv d\left(P_{j}, R_{h}\right)$ denotes the distance between city $j$ and landfill $h$.

Equation (6) is the first-order condition with respect to $Y_{0}$ and states the equality between marginal cost and marginal gain of an increase in $Y_{0}$ (where, in this framework, a gain means a reduction in total cost). Concerning the marginal cost (left-hand side), the first term is the marginal construction cost, 
the second term comes from the fact that, by increasing the lifetime $T_{1}$, the transportation costs $T C_{0}$ will have to be paid for a longer time and the third comes from the interaction between capacity and location. If landfills 0 and $k$ (for some $k>0$ ) are 'as-close-as-possible', then an increase in $Y_{0}$ enlarges the safety region around $R_{0}$ and reduces the space available to locate landfill $k$, which will have to move to a different location, and thereby possibly increase transportation costs. The term $\beta \lambda_{0 i}$ can be interpreted as the shadow price or scarcity cost of land (or space) between landfills 0 and $i$, i.e., the marginal reduction in total cost if landfills 0 and $i$ would come marginally closer to each other. This cost shows up only if landfills 0 and $i$ are 'as-close-as-possible' (see Figure 1). Otherwise, if would be feasible for the planner to move them closer. If these landfills are not moved, then the reason is that no gain (in the form of cost reduction) would result. Mathematically, if landfills 0 and $i$ are not 'as-close-as-possible', then $\lambda_{0 i}=0$ holds, meaning that land scarcity turns out not to be an economic problem involving landfills 0 and $i$. The total marginal cost linked to this third effect is given by $\Lambda_{0} \equiv \beta \sum_{i \in A_{0}} \lambda_{0 i}$, where

$$
A_{0} \equiv\left\{i=1, \ldots, K-1 / d\left(R_{i}, R_{0}\right)=\beta\left(Y_{i}+Y_{0}\right)\right\}
$$

is the set of landfills which are 'as-close-as-possible' to landfill 0 . In the same way, $\Lambda_{i}$ and $A_{i}$ can be defined for any $i=1, \ldots, K-1$. The term $\Lambda_{i}$ can be interpreted as the shadow price or scarcity price of land around landfill $i$. The marginal gain of an increase in $Y_{0}$ (first term on the right-hand side of equation (6)) comes from the fact that a longer lifetime defers all the construction and transportation costs of future landfills. Given the time preference, as measured by $\delta$, this results in a smaller discounted cost. As for the rest of the terms in (6), $\mu$ measures the marginal increment in setup costs, which results from a marginal increment in the total amount of waste to be landfilled, $\tau Q$. If an excess capacity exists, i.e. $\tau Q<\sum_{i=0}^{K-1} Y_{i}$, then (9) implies that this impact equals zero because, in this case, more waste does not imply that more installed capacity is needed. Multipliers $\alpha_{00}$ and $\alpha_{01}$ account for the possibility of minimum and maximum capacity constraints being binding. The same interpretation applies for conditions ( 7 ) linked to $Y_{h}, h=1, \ldots, K-1$. For the final landfill, the effect of deferring future costs is not present, because no future exists ${ }^{6}$.

The location conditions (8) result from equating the derivatives of $\mathcal{L}$ with respect to $r_{h 1}$ and $r_{h 2}$ to zero, and the interpretation is the following ${ }^{7}$. Moving (marginally) a landfill $h$ has two effects: the first one is related to the distance from the cities and to transportation costs. An increment in, say, $r_{h 1}$ increases (decreases) the distance between landfill $h$ and city $j$ if $r_{h 1}>p_{j 1}\left(r_{h 1}<p_{j 1}\right)$, and this effect needs to be accrued across all cities. The distance from some cities may increase and from others may decrease, and hence some terms in $\sum_{j=1}^{m} q_{j} \frac{p_{j 1}-r_{h 1}}{d_{j h}}$ may be positive and others negative. The second effect accounts for the fact that, if landfills $h$ and $i$ are 'as-close-as-possible', then a marginal change in the location of landfill $h$ may require a movement for landfill $i$ and thereby affect transportation costs.

These two location effects can be seen in Figure 2. The optimal location of landfill $h$ (i.e., the optimal value of $r_{h 1}$ and $r_{h 2}$ ) must be chosen by considering the location of two cities (points $P_{1}$ and $P_{2}$ ) and another landfill $i$ (point $R_{i}$ ). When determining $r_{h 1}$ (horizontal coordinate), one should take into account that $p_{11}-r_{h 1}<0$ and $p_{21}-r_{h 1}<0$. Hence, starting from the position in the figure, if $R_{h}$ is marginally moved to the right (increase $r_{h 1}$ ), then the distance with respect to city 1 (2) will increase (decrease) as will the associated transportation cost. The marginal effect on transportation costs (either an increase or a decrease) will be given by the (discounted value of the) weighted aggregation $q_{1} \frac{p_{1}-r_{h 1}}{d_{1 h}}+q_{2} \frac{p_{21}-r_{h 1}}{d_{2 h}}$. On the other hand, if landfills $i$ and $h$ are 'as-close-as-possible', and $r_{i 1}>r_{h 1}$, then an increase in $r_{h 1}$ is not feasible unless $R_{i}$ also moves or the capacities are adjusted to maintain feasibility. This will imply a 
cost increment measured by the shadow price $\lambda_{h i}$. If landfills $i$ and $h$ are not 'as-close-as-possible', then $\lambda_{h i}=0$ and the latter effect does not show up.

By manipulating condition (7) for two consecutive landfills and assuming that minimum and maximum capacity constraints are not binding, the following first-order difference equation relating $Y_{h}$ and $Y_{h+1}$ is obtained:

$$
C^{\prime}\left(Y_{h}\right)=e^{-\frac{\delta}{Q} Y_{h}}\left[C^{\prime}\left(Y_{h+1}\right)+\frac{\delta}{Q} C\left(Y_{h+1}\right)+\frac{\left(T C_{h+1}-T C_{h}\right)}{Q}\right]+e^{\frac{\delta}{Q} \sum_{\ell=0}^{h-1} Y_{\ell}}\left[\Lambda_{h+1}-\Lambda_{h}\right] .
$$

This expression can be considered as a generalization of the so-called Optimal Capacity Condition presented by André and Cerdá $(2001,2004)$ in the sense that not only does it refer to optimal capacity, but also to location. If location is not a problem, then the scarcity cost of land becomes zero, $\Lambda_{h+1}=\Lambda_{h}=0$, and (13) collapses to basically the same condition as that presented in Andre and Cerdá (2001, Proposition $1)^{8}$.

Equation (13) can be seen as a no-arbitrage condition which rules out any gain (i.e., any reduction in total cost) in transferring some capacity from landfill $h$ to landfill $h+1$ or vice versa. If we disregard the last term (assuming $\left.\Lambda_{h+1}=\Lambda_{h}\right)$ and there is no time preference $(\delta=0)$ then the following simpler condition is obtained:

$$
C^{\prime}\left(Y_{h}\right)+\frac{T C_{h}}{Q}=C^{\prime}\left(Y_{h+1}\right)+\frac{T C_{h+1}}{Q}
$$

which means that the marginal cost of capacity in landfill $h$ (marginal construction cost plus transportation cost per unit of waste) must equal that of landfill $h+1$. If $\delta \neq 0$, the marginal cost in period $h+1$ must be discounted and the effect of deferring future costs must be considered. The last term in (13) accounts for the different value of land around landfills.

Rewriting condition (13) yields a difference equation for the evolution of the scarcity cost of land from landfill $h$ to $h+1$.

$$
\Lambda_{h+1}-\Lambda_{h}=e^{-\frac{\delta}{Q} \sum_{\ell=0}^{h-1} Y_{\ell}}\left\{C^{\prime}\left(Y_{h}\right)-e^{-\frac{\delta}{Q} Y_{h}}\left[C^{\prime}\left(Y_{h+1}\right)+\frac{\delta}{Q} C\left(Y_{h+1}\right)+\frac{\left(T C_{h+1}-T C_{h}\right)}{Q}\right]\right\} .
$$

According to (14), the scarcity cost of landfill $h+1$ is larger (as compared to that of landfill $h$ ) the higher the marginal construction cost of landfill $h$, the lower the (total and marginal) construction cost of landfill $h+1$, and the lower the transportation cost per unit of waste associated to landfill $h+1$ as compared to that associated to landfill $h$. Summing up, the space around landfill $h$ is more valuable (and hence, its shadow price is higher) the higher the cost advantage (or the lower the disadvantage) of landfill $h+1$ (given the place where it is located and its capacity) as compared to landfill $h$.

The time pattern of the shadow price of land (i.e., the sign of $\Lambda_{h+1}-\Lambda_{h}$ ) is inherently determined by two underlying effects which we can label as "proximity effect" and "scarcity effect". The proximity effect is narrowly linked to time preference and it causes the sequence of $\Lambda$ to decline. As discussed in section 3.4, landfills are typically used in increasing order of distance from the cities, i.e., the first landfill is normally located as close as possible to the waste generators in order to save transportation cost. When the closest region is no longer available, the second landfill is located as close as possible within the remaining region and so on. This means that the weighted distance, or equivalently the instantaneous transportation costs $T C_{i}$, is typically increasing in $i$. In turn, this implies that space is more valuable around the first landfills, which are closer to the cities and have a higher weight in the objective function. The scarcity effect goes in the opposite direction. Assume a situation in which the available land is already full of landfills, becoming 
really scarce. As time goes by, it will be harder and harder to find a place to locate an additional landfill. This effect will push the shadow price upwards and, if it happens to dominate the proximity effect, it will lead to a net raise in $\Lambda$.

If space scarcity does not become a relevant force within the planning horizon, the scarcity effect will not show up. The typical implication is then a declining sequence of $\Lambda$. If, on the other hand, space turns out to be significantly scarce, the second effect will naturally show up for the last landfill(s) and not for the first part of the sequence (when more space is available). This situation may result in a $U$ shape for the $\Lambda$ sequence. These results are illustrated in the examples displayed in section 4 .

\subsection{Minimum and maximum number of landfills}

The minimum capacity constraint, together with the fact that $\Omega$ is bounded, guarantee that $K$ is finite. Proposition 1 states lower and upper bounds for $K$.

Proposition 1 The number of optimal landfills in problem (1) is bounded by:

$$
\Psi\left(\frac{\tau Q}{\bar{Y}}\right) \leq K \leq \Psi\left(\frac{\tau Q}{\underline{Y}}\right)
$$

where

$$
\Psi(x) \equiv \begin{cases}x & \text { if } x \text { is an integer } \\ \operatorname{Int}(x+1) & \text { otherwise }\end{cases}
$$

and the operator Int denotes the integer part.

\section{Proof See Section 6.1}

If condition $\sum_{i=0}^{K-1} Y_{i} \geq \tau Q$ in problem (5) is replaced with $\sum_{i=0}^{K-1} Y_{i}=\tau Q$ (which means that excess capacity is ruled out), then the second part of proposition 1 is transformed into $K \leq \operatorname{Int}\left(\frac{\tau Q}{\underline{Y}}\right)$. To understand this, note that $Y \geq \underline{Y}$ implies $K \leq \frac{\tau Q}{\underline{Y}}$, however if $\frac{\tau Q}{\underline{Y}}$ is not an integer, then $K=$ Int $\left(\frac{\tau Q}{\underline{Y}}+1\right)$ is not feasible because $\sum_{i=0}^{K-1} Y_{i} \geq K \underline{Y}>\tau Q$, and hence the maximum feasible number of landfills is $\operatorname{Int}\left(\frac{\tau Q}{\underline{Y}}\right)$.

Define the indirect cost function for $K$ landfills as $\mathcal{J}_{K}(\Theta) \equiv \min _{\{Y, R\}} J(K, Y, R, \Theta)$, where $\Theta$ denotes the set of parameters of the problem, including $\tau, \phi, \delta, \beta, Q, \underline{Y}, \bar{Y}$. The following proposition states the impact of any of these parameters on the indirect cost function.

Proposition $2 \mathcal{J}_{K}(\Theta)$ is non-decreasing in $\tau, \phi, \beta, Q, \underline{Y}$ and non-increasing in $\delta$ and $\bar{Y}$.

\section{Proof See Section 6.2}

\subsection{Discussion of excess capacity}

Condition $T_{K} \geq \tau$ in problem (1), or alternatively $\sum_{i=0}^{K-1} Y_{i} \geq \tau Q$ in problem (5), allow for the possibility of an excess capacity, in such a way that when time $\tau$ is reached there is some capacity that remains unexhausted. When this is the case, a subtle remark should be made about the interpretation of $T_{K}$. Since we assume that the capacity of landfills $0,1, \ldots, K-2$ is always fully depleted, then $T_{i+1}-T_{i}$ is, at the same time, the physical lifetime and the economic lifetime of landfill $i$ (for $i=0, \ldots, K-2$ ). Nevertheless $T_{K}$ can be interpreted as the time at which the capacity of landfill $K-1$ would become 
depleted if it were used at the current rate. Therefore, $T_{K}-T_{K-1}$ can be seen as the physical lifetime of landfill $K-1$. If there is (there is no) excess capacity, it will not (it will) coincide with its economic lifetime $\tau-T_{K-1}$.

Since construction cost increases with capacity, it seems unreasonable that a rational decision-maker could be willing to incur such an excess capacity. In André and Cerdá (2004) (proposition 1) it is shown that this counter-intuitive result may arise when dealing with landfill construction and a necessary and sufficient condition is provided for this result to happen, in a context where all the landfills are constrained to have the same capacity. Excess capacity seems even more surprising in this model than in the one in André and Cerdá (2004) for two reasons: firstly, space scarcity implies additional cost in terms of wasted space, coming from constructed but unexhausted capacity. Secondly, unlike the case studied in André and Cerdá (2004), in this paper the capacity of landfills is assumed to be variable, so it is possible, in general, to increase the capacity of the first landfill(s) in order to delay future costs, and then decrease that of the final landfill(s) to avoid excess capacity and reduce total cost. Nevertheless, we argue that excess capacity can also result in this case. Specifically, there is a particular situation where some excess capacity can arise in a natural way; namely, when the lower capacity constraint is binding for the final landfill(s).

First, one should bear in mind that, since the planner's objective is to minimize costs, it is never optimal to under-exhaust the capacity of any landfill $h(h=0, \ldots, K-2)$ different from the final one, since total discounted cost could be reduced simply by exhausting landfill $h$ and delaying the costs of landfills $h+1, \ldots, K-1$. Nevertheless, this is not always the case for the final landfill. The simplest case is that in which $Q \tau<\underline{Y}$. In this case, even the capacity of the smallest feasible landfill is too large to meet the requirements in the planning horizon. In this case, the solution implies $K=1$ and $Y_{0}=\underline{Y}>\tau Q$. Assume now that $K=2$. If we set $Y_{0}=Q \tau-\underline{Y}, Y_{1}=\underline{Y}$, and choose locations $R_{0}, R_{1}$ consistent with $Y_{0}$ and $Y_{1}$, then a feasible solution is found without excess capacity. Assume, moreover, that the derivative of the Lagrangian with respect to $Y_{0}$, evaluated at $Y_{0}, Y_{1}$ is negative, i.e.,

$$
\begin{array}{r}
C^{\prime}\left(Y_{0}\right)+e^{-\frac{\delta}{Q}\left(Y_{0}\right)} \frac{T C_{0}}{Q}+\beta \lambda_{01}< \\
\frac{\delta}{Q} e^{-\frac{\delta}{Q}\left(Y_{0}\right)}\left[C\left(Y_{1}\right)+\left(1-e^{-\frac{\delta}{Q} Y_{1}}\right) \frac{T C_{1}}{\delta}\right]+\mu+\alpha_{00}-\alpha_{10} .
\end{array}
$$

In this case, total discounted cost could be reduced by increasing $Y_{0}$. This would result in an overall excess capacity that could not be eliminated by reducing $Y_{1}$, which is already at its lower bound. The principal concept behind (15) is that, if marginal cost is "low enough", a small increment in the capacity of the first landfill would result in a small cost increment that could be more than compensated by the discounted cost reduction as a consequence of postponing the construction of future landfills.

In general, assume a modified version of problem (5), subject to $\sum_{i=0}^{K-1} Y_{i}=\tau Q$ (with strict equality rather than inequality) is solved. Denote the optimal solution of this problem as $S^{e q} \equiv\left(Y^{e q}, R^{e q}\right)$, where $e q$ stands for equality. Denote $\left.\frac{\partial \mathcal{L}}{\partial Y_{h}}\right|_{s^{e q}}$ as the derivative of the Lagrangian with respect to $Y_{h}$ evaluated at $S^{e q}$. If the following condition holds

$$
\left.\frac{\partial \mathcal{L}}{\partial Y_{h}}\right|_{s^{*}}<0
$$

for any $h=0,1, \ldots, K-2$, then this means that, starting from a situation without excess capacity, increasing the capacity of landfill $h$ would result in a reduction of cost. This implies that it would be 
optimal to incur an excess capacity if this excess capacity could not be compensated for feasibility reasons. The interpretation of (16) is basically the same as that of (15).

This result is illustrated in a numerical example in the Appendix (Section 6.4). This example shows that, in a solution $\left\{R^{*}, Y^{*}\right\}$ with optimal excess capacity, the capacity of the final landfill(s) is equal to the lower bound. This typically happens when there is some excess capacity. The following property shows that, if some excess capacity exists, the minimum capacity constraint binds for at least the final landfill. The idea is that, for the final landfill, there is no gain in increasing the capacity, since future costs cannot be delayed, as no future exists.

Property 1. In the solution to problem (5), if $\sum_{i=0}^{K-1} Y_{i}>\tau Q$, then it must be the case that $Y_{K-1}=\underline{Y}$.

\section{Proof See Section 6.3.}

The idea behind this result is the following. Consider a feasible solution $\{K, Y, R\}$ with excess capacity, meaning that there is more installed capacity than needed for the whole amount of waste in the planning horizon. Assume now that the solution is altered just by reducing the capacity of the final landfill, $Y_{K-1}$, until the excess capacity disappears. This change would unambiguously reduce the discounted cost because the construction cost of the final landfill would decrease and everything else would remain unchanged. Therefore, if $\{K, Y, R\}$ is a cost-minimizing solution, reducing $Y_{K-1}$ can not feasible or, in other words, $Y_{K-1}=\underline{Y}$. One conclusion that can be immediately drawn is that an excess capacity can never occur if there is no lower bound for capacity. This is formalized in the following corollary:

Corollary 1 If $\underline{Y}=0$, in the solution to problem (5) it must hold that $\sum_{i=0}^{K-1} Y_{i}=\tau Q$, i.e., there is no excess capacity.

Proof Immediately follows from Property 1.

\subsection{Discussion about location and optimal order of landfills}

Once the optimal number of landfills $K^{*}$, the optimal capacities $Y^{*}$, and the optimal locations $R^{*}$ have been determined, and since $\left\{K^{*}, Y^{*}, R^{*}\right\}$ is feasible by definition, then any solution $\left\{K^{*}, \tilde{Y}, \tilde{R}\right\}$, where $\tilde{Y}$ is a permutation of the elements of $Y^{*}$ and $\tilde{R}$ is the associated permutation of $R^{*}$, would yield a feasible (but not necessarily optimal) solution. Note that re-ordering the locations without re-ordering the capacities may not be feasible. It is relevant to study the optimal order in which landfills should be used, once we know their capacity and location.

A number of landfills can be conceptualized as several deposits of a natural exhaustible resource. A classic result by Herfindahl (1967) states that several deposits of a natural resource should be exploited in an increasing order of marginal extraction costs. André and Cerdá (2001) show that Herfindahl's result holds for landfill construction in the sense that, if the only difference between the places available for landfill location is transportation cost, then it is optimal to use such places beginning with the one with the lowest cost and following in the order of increasing unit cost. In the case of a single city, this result implies that landfill $i$ should be closer to the city than landfill $j$, for any $j>i$. The equivalent result in our model would imply the weighted distance, or equivalently the instantaneous transportation costs $T C_{i}$, to be increasing in $i$.

Nevertheless, Hartwick, Kemp and Long (1986) demonstrated that Herfindahl's result does not necessarily hold when each site has different setup costs. Our results are consistent with this observation since 
our model includes setup costs. Moreover, we present a different version of the study by Hartwick, Kemp and Long in the sense that setup costs are endogenous and depend on capacity. As a result, we conclude that, for those landfills whose capacity is optimally the same, it is always optimal to use first the closest (lowest cost) places and then move to the further (higher cost) locations, and hence Herfindahl's result holds. As a matter of fact, this result is consistent with the evidence that, as time goes on, landfills are normally constructed further away from cities and this is consistent with most of the numerical exercises performed (see Section 4). Nevertheless, it is not trivial to conclude that Herfindahl's result always holds in our framework because landfills may differ due to their location and their capacity ${ }^{9}$ and capacity, in turn, determines the lifetime of landfills. Henceforth, reordering the sequence of capacities affects total (discounted) cost and this effect may be strong enough to offset the effect linked to transportation costs. As a consequence, no general statement can be made regarding the order of $T C_{i}$. Proposition 3 shows that $T C_{i}$ always increases with $i$ if the sequence of capacities is constant.

Proposition 3 In the optimal solution for problem (1), if $Y_{i}=Y_{j}$ holds for a pair of landfills $i, j$, such that $i<j$, then $T C_{i} \leq T C_{j}$.

Proof The objective function of (1) can be written as $\sum_{i=1}^{K-1} \gamma_{i}\left(C\left(Y_{i}\right)+\frac{T C_{i}}{\delta}\left(1-e^{-\delta \frac{Y_{i}}{Q}}\right)\right)$, where $\gamma_{i} \equiv$ $e^{-\delta T_{i}}$ and $\gamma_{i}>\gamma_{j}, \forall i>j$. Assume the optimal solution is given by $\left\{K^{*}, Y^{*}, R^{*}\right\}$, such that $Y_{i}=Y_{i+1}$, $T C_{i}>T C_{i+1}$ for some $i=0, \ldots, K-2$. Consider the alternative solution $\left\{K^{*}, \tilde{Y}, \tilde{R}\right\}$ where $\tilde{Y}$ is constructed by shifting the positions of landfills $i$ and $i+1$ and where $\tilde{R}$ is the associated permutation of the elements of $R^{*}$, while keeping the rest of the elements in $\left\{K^{*}, Y^{*}, R^{*}\right\}$ unchanged. Clearly $\left\{K^{*}, \tilde{Y}, \tilde{R}\right\}$ is feasible and provides a smaller discounted cost than $\left\{K^{*}, Y^{*}, R^{*}\right\}$, therefore $\left\{K^{*}, Y^{*}, R^{*}\right\}$ cannot be a solution for (1).

In the proof of proposition 3 we can see why the condition of constant capacity is required. If $Y_{i} \neq Y_{i+1}$, then shifting $Y_{i}$ and $Y_{i+1}$ results in different values for $\gamma_{i+1}, C\left(Y_{i}\right)$ and $C\left(Y_{j}\right)$, and hence no general statement can be made. Using a continuity argument, it can be concluded that if $Y_{i}$ and $Y_{j}$ are close enough, for some $i<j$, then $T C_{i} \leq T C_{j}$ must also hold. As a matter of fact, in the numerical exercises performed, it is found that $T C$ increases in most cases regardless of the order of the capacities, although some counter-examples exist, such as the one presented in the Appendix (section 6.5).

\section{Numerical illustration}

The complexity of the problem and its non-convex nature prevent us from attaining an analytical solution and some numerical method is therefore needed. To overcome the difficulty of dealing with several local minima, global optimization techniques are used. A basic reference for most aspects of global optimization can be found in Horst and Pardalos (1995). See also Neumaier (2001) for the state of the art. See Hansen et al. (1995) for a survey of applications to facility location. In our case, a global optimization problem is solved for every possible value of $K$ and that which provides minimum discounted cost is chosen.

We are not particularly interested in the numerical values, but rather in the properties of the solution and how different parameters influence its behavior. Therefore, an example starting from an arbitrary set of parameter values (with no particular numerical meaning) is shown and some sensitivity analysis is performed. For every problem, we use a radial basis function algorithm implemented in the optimization environment Tomlab for Matlab (see Holmstrom, 1999 and Gutmann, 2001) and a genetic algorithm 
implemented in Mathematica 5.0. When both methods yield different solutions, we choose the best one (that one giving a lower cost). The search is refined around the optimum using the Newton algorithm.

Assume the construction cost function is linear: $C(Y)=a+b Y$, $a$ representing some fixed cost and $b$ marginal construction cost. There are five cities located at points $(0,0),(1,0),(1,1),(0,1)$ and $(2,2)$, generating flows of waste $3,4,2,1$ and 3 , such that $Q=13$. The feasible region is given by the rectangle defined by the extreme points $(2,3),(17,16)$. To guarantee that the safety regions of all the landfills fall within the feasible region, the constraints $2+\beta Y_{i} \leq r_{i 1} \leq 17-\beta Y_{i}, 3+\beta Y_{i} \leq r_{i 2} \leq 16-\beta Y_{i}$, for $i=0, \ldots K-1$ are added. The following benchmark values for the parameters are set:

$$
\begin{aligned}
& a=1000 \quad \underline{Y}=90 \quad \beta=0.01 \quad \delta=0.05 \\
& b=10 \quad \bar{Y}=400 \quad \phi=1 \quad \tau=56
\end{aligned}
$$

From proposition 1, it is known that the number of landfills is bounded by $2 \leq K \leq 9$. The global optimization problem is numerically solved and the optimal number of landfills is found to be $K^{*}=4$, the optimal locations $R^{*}=\{(3.6,4.6),(7.0,4.8),(3.9,8.1),(7.8,8.5)\}$ and the optimal capacities $Y^{*}=\{163$, 176, 189, 200\}. The solution is illustrated in Figure 4. The left panel displays the location of landfills. The triangles represent cities and the squares represent landfills. The order of landfills is indicated by numbers. The feasible region is delimited by the white rectangle. The right-hand panel shows the sequence of capacities. There is no excess capacity and the sequence of capacities increases, however, as shown bellow, no general conclusion can be drawn about this sequence increasing or decreasing. The instantaneous transportation costs associated with landfills are given by $T C_{0}=62, T C_{1}=95, T C_{2}=104, T C_{3}=135$, and hence they are increasing, as is typically the case. Furthermore, the shadow price of land around each landfill can be calculated as $\Lambda_{0}=0.96, \Lambda_{1}=0.74, \Lambda_{2}=0.44, \Lambda_{3}=0.23$. Since these shadow prices are declining we can conclude that the sequence is dominated by the proximity effect (see discussion in section 3.1).

Some sensitivity analysis exercises starting from the benchmark parameter values in (17) are performed and the most interesting results are shown. The location and the order of landfills is primarily determined by the position of cities. As an illustration, assume that the location of the second city is moved from the point $(1,0)$ to the point $(1,10)$, while the rest of the settings remain unchanged. The new solution, illustrated in Figure 5 , is given by $K^{*}=4 ; R^{*}=\{(3.7,4.7),(3.8,8.3),(7.3,4.8),(7.5,8.5)\} ; Y^{*}=\{175,184$, $182,187\}$. Note that the locations of the landfills are very similar to those in the benchmark case, but the order is different. The second landfill is now the one closest to the city at $(1,10)$ in order to minimize total discounted cost. Observe also that, in this case, the sequence of capacity is not monotonically increasing nor decreasing. This feature depends on the specific combination of the parameter values and no general statement can be made.

An increment in parameter $a$ makes the construction of any landfill more expensive irrespective of its capacity. As a consequence, when $a$ increases enough, a reduction in the number of landfills and an increase in their average capacity become the optimal solution, in order to avoid incurring a large fixed cost many times. See the left-hand panel of Figure 6 . Another interesting result is that increasing $a$ makes the sequence of capacities decrease more, i.e., the capacity of the first landfills become larger and that of the last landfills become smaller. As the setup cost $(a)$ escalates, it becomes more economical to increase the capacity of the first landfill(s) in order to postpone the construction of future landfills. Since $a$ is a fixed cost, it does not affect the impact of capacity on total cost. See the right-hand panel of Figure 6 . To appreciate the effect on the sequence of capacities, a range of $a$ for which $K^{*}$ is constant is shown (in this 
case, $\left.K^{*}=3\right)$. It is interesting to note that there is a trade-off when increasing the capacity of the first landfill(s): on the one hand, the construction of the subsequent landfills will need to be undertaken later (reducing discounted cost). On the other hand, the safety region around the first landfill(s) increases, so that the locations of the subsequent landfills are displaced further away from the cities, implying higher transportation costs.

Consider now that, during the planning horizon, a suffers a sudden large increase. The planned sequence of landfills is therefore no longer optimal and a new policy needs to be calculated instead. In the new sequence, the number of landfills is smaller and the average capacity of landfills is larger. A probable and reasonable explanation for the observed trends in the number and capacity of landfills discussed in the introduction (small landfills have closed and large landfills have notably grown in number and size, see US EPA, 1988; Repa, 2000) is that the fixed construction cost of landfills has greatly increased owing to the scarcity of land and tougher environmental and technical regulations. This fact is illustrated in Figure 7. The dashed line denotes a sequence of optimal capacities for a given value of $a$. At time $t_{s}$, parameter $a$ suddenly increases by a large amount. The old sequence is no longer optimal and a new problem (starting at $t_{s}$ ) must be solved to find a new optimal sequence of capacities (represented by the solid line). According to our model, this shock causes the average capacity of landfills to increase and the optimal number of landfills to decrease.

Parameter $b$ has exactly the opposite effect: as $b$ increases, it is more costly to construct large landfills, and hence it becomes profitable to build many small landfills. Therefore, $K^{*}$ turns out to increase with $b$. As landfills become smaller, so do their safety regions and, therefore, the distances between the landfills, as well as their proximity cities where the waste is generated become closer. It is also interesting to note that, as $b$ increases, the capacity of the first landfill(s) tends to decrease and that of the final landfill(s) tends to increase, since a larger capacity now implies a larger construction cost, and that effect is more important for the initial landfills given the time preference (see illustration in Figure 8).

Figure 9 illustrates the impact of $\tau$ on the optimal number of landfills $K^{*}$ (left-hand panel) and the average capacity $Y_{\text {mean }}=\sum_{i=0}^{K-1} Y_{i} / K$ (right-hand panel). An increment in parameter $\tau$ implies a larger overall amount of waste to be landfilled $(Q \tau)$. Feasibility requires either an increase in the number or the capacity of landfills. "Small" increments of $\tau$ lead to an increase in the average individual capacity and keep $K^{*}$ unchanged, up to the point where the increase of $\tau$ is large enough to cause a new landfill to be profitable, which allows a reduction in average capacity. Therefore, as a function of $\tau, K^{*}$ displays a stair shape and $Y_{\text {mean }}$ displays a sawtooth shape.

To save space, a brief summary of the results concerning the rest of the parameters in (17) is presented. Increasing the discount rate $\delta$ makes the optimal number of landfills increase and the sequence of capacities decrease more, since the costs associated to the first landfill become more important for the objective function. Increasing parameter $\phi$ causes the optimal number of landfills to increase and their capacity to decrease, which makes the landfills closer and reduces transportation costs. The effect of parameter $\beta$ is rather predictable, as it makes the safety regions around landfills increase and so they become more distant one from another. This reduces the feasible region and the problem may ultimately become infeasible if $\beta$ increases sufficiently. Changes in parameters $\underline{Y}$ and $\bar{Y}$ only become relevant when the lower and upper capacity limits are binding. The effect is trivial for capacity (i.e., if the lower capacity constraint is binding for some landfills and $\underline{Y}$ increases, then the capacity of such landfills has to increase and so on), and the locations optimally adjust to these changes. 
The example discussed so far may seem somehow special since there are very little distances between the cities. A different example is now shown in order to illustrate that all the basic intuitions hold when the cities are located in a different way. Assume there is the largest city (say, the capital), located at point $(1,1)$, which generates an instantaneous amount of waste equal to 8 . This city is represented in Figure 10 by a larger triangle. The rest of the (smaller) cities are located at points $(8,0),(1,10),(8,18)$, $(18,10)$ and the associated flows of waste are $2,1,1,1$. The other parameter values are:

$$
\begin{aligned}
& a=3900 \quad \underline{Y}=90 \quad \beta=0.01 \quad \delta=0.05 \\
& b=10 \quad \bar{Y}=400 \quad \phi=1 \quad \tau=100
\end{aligned}
$$

The solution (see Figure 10) is given by $K^{*}=4, R^{*}=\{(5.2,6.2),(11.58,6.17),(5.29,12.7),(11.9,12.67)\}$ and the optimal capacities $Y^{*}=\{320.61,317.45,328.98,332.94\}$. In this case, given the disperse location of the cities, it is not accurate to say that the distance from landfills to cities increases over time, since distance increases with respect to some cities and decreases with respect to others. The instantaneous transportation costs associated with landfills are given by $T C_{0}=98.28, T C_{1}=139.63, T C_{2}=149.70$, $T C_{3}=178.81$. This sequence also increases, as is typically the case. This fact illustrates that the relevant piece of information when dealing with several cities is the weighted distance from all the cities or, in other words, the aggregated transportation cost. Similarly, the shadow price of land around each landfill is given by the declining sequence $\Lambda_{0}=0.47, \Lambda_{1}=0.39, \Lambda_{2}=0.14, \Lambda_{3}=0.06$.

In the examples presented so far, land scarcity does not appear to be a relevant force to determine the evolution of the shadow price, since the sequence of $\Lambda$ is shown to be monotonically decreasing. This situation may change if the time horizon (and, therefore, the total amount of waste to be landfilled) increases while keeping the whole feasible region unchanged. Assume an alternative version of example (18) with $\tau=108.2$ and the other parameter values unchanged. The optimal number of landfills is $K^{*}=6$ and the sequence of scarcity prices is $\Lambda_{0}=0.48, \Lambda_{1}=0.39, \Lambda_{2}=0.15, \Lambda_{3}=0.05, \Lambda_{4}=0.01, \Lambda_{5}=0.12$. In this problem, land scarcity becomes relevant for the last part of the sequence of landfills. Therefore, the proximity effect dominates at the beginning but the scarcity effect dominates at the end, and the sequence of scarcity prices displays a U shape.

\section{Conclusions and discussion}

We have presented a spatial and sequential model to study the joint determination of the optimal capacity and location of landfills and shown how these decisions interact with each other. The capacity decision has spatial implications since the capacity of a landfill affects the feasible region for the rest of the landfills, and temporal implications, because the capacity determines the lifetime of the landfill and hence the instant of time when the next landfills will need to be constructed. Because of the dynamic nature of the problem, the whole sequence of optimal capacities and locations must be jointly decided. In other words, it is not optimal to design a single landfill or, in general, to solve independently the problem for each subinterval.

Concerning the capacity decision, this can be seen as a dynamic problem involving the sequential exploitation of several deposits of a resource, with the peculiarity that capacity is an endogenous decision. The Optimal Capacity Condition expresses the impossibility of reducing cost, in the minimum cost solution, by transferring capacity from one landfill to another when the location problem is taken into account. Increasing the capacity of any landfill increases its construction costs as well as its lifetime. 
Moreover, it affects the location possibilities for the rest of landfills, particularly for those that are 'asclose-as-possible' to it. All these influences result in different marginal cost effects, which must cancel each other out in the optimal sequence of capacities.

From the point of view of location, the problem can be seen as siting a number of facilities that compete for limited space, and similar intuitions apply to any facilities other than landfills. Any marginal spatial movement of a facility should take into account the effects on the distance to the consumers and the position with respect to the competing facilities. The structure of the problem allows an explicit measure of the scarcity value of land to be attained, which varies across different areas. A difference equation for the evolution of this scarcity value is also obtained. In general, this scarcity value is driven by a proximity effect and a scarcity effect. The former is typically dominating at the beginning of the sequence of landfills whereas the latter may become relevant at the end, when there is less space available. We have shown that, if the scarcity effect is strong enough, the shadow price of land may happen to display a U shape.

In some special cases, the economic lifetime of the last landfill in a sequence could be different from the physical lifetime. In other words, it may be optimal to set up an excess capacity if the marginal construction cost of any landfill in the sequence is overcompensated by the reduction in total discounted cost achieved by deferring the construction of future landfills. This result implies that the lower capacity constraint is binding for at least the last landfill of the sequence.

Given a feasible sequence of locations and capacities, any permutation is also feasible. Typically, landfills are used in increasing order of distance from the cities, which is consistent with the observed trends in practice. Nevertheless, this result can fail to hold in theory if the optimal sequence of capacities is not constant.

We have illustrated the use of global optimization methods to find the solution in a specific example with a linear construction cost function. From the sensitivity analysis performed we know that a larger fixed construction cost results in optimally decreasing the number of landfills and making the sequence of capacities decrease more (or increase less). If marginal construction cost increases, the optimal number of landfills increases and the optimal sequence of capacities increases more (or decreases less). When the time horizon varies, the optimal number of landfills behaves as a stair-shaped function and the average capacity displays a sawtooth shape.

This analysis can be extended and enriched in several ways. First, it is relevant to note that over recent decades, the use of the term waste has been somewhat modified to account for differences in origin (domestic, industrial), toxicity and environmental damage (hospital, radioactive, etc.). On the other hand, there is nowadays an increasing interest in recycling as well as tougher regulations regarding waste management practices. Therefore, it would be interesting to study the joint decision of landfilling and recycling. Apart from the setting up of disposal (and perhaps recycling) facilities, societies have to decide which proportion of waste to manage with each treatment method. Obviously, these decisions interact with those of capacity and location of waste facilities. Moreover, the fact that the flow of waste may be variable (as has been historically the case in practice), introduces a new dynamic element into the problem.

An additional plausible extension of this analysis is to allow for the possibility of several landfills to operate simultaneously, where a further decision would be the amount of waste to be sent to each open landfill. This possibility has been explored by Gaudet et al. (2001), but in that work, the location and capacity of all the landfills is given and the only decisions to be made are related to the optimal time 
to open each landfill and the optimal distribution of waste to each landfill. A notably more complex and challenging problem would result by letting the location and capacity of landfills be endogenous.

\section{Notes}

${ }^{1}$ The U.S. Environmental Protection Agency (EPA) defines a landfill as "An engineering method of disposing of waste on land in an environmentally acceptable manner". See EPA golssary at

http://www.environment.nsw.gov.au/mao/glossary.htm\#l

${ }^{2}$ For example, Shulman, 1991, uses Lagrangian relaxation while Fong and Srinivasan, 1986, develop a heuristic algorithm.

${ }^{3} C(Y)$ can be thought of as measuring the present value of both construction and closure costs. If $G_{1}(Y)$ denotes construction and $G_{2}(Y)$ closure cost of a landfill with capacity $Y$ and lifetime $[0, T)$, total cost is given by $G(Y, T) \equiv G_{1}(Y)+e^{-\delta T} G_{2}(Y)$. Once $Y$ is decided, and since the flow of waste is assumed to be exogenous, $T$ can be expressed as a function $T(Y)$ and $G(Y, T)$ collapses to a function depending only on $Y$ and the parameters of the model:

$$
G(Y, T) \equiv G_{1}(Y)+e^{-\delta T} G_{2}(Y)=G_{1}(Y)+e^{-\delta T(Y)} G_{2}(Y) \equiv C(Y)
$$

where $\delta$ is the discount rate.

${ }^{4}$ André and Cerdá (2001) present a case with time-varying waste $Q(t)$. Solving the integral $\int_{T_{i}}^{T_{i+1}} Q(t) d t$ yields a condition such as $Y_{i}=F\left(T_{i}, T_{i+1}\right)$ or, solving for $T_{i+1}$, a condition such as $T_{i+1}=\Phi\left(T_{i}, Y_{i}\right)$, which can be seen as a state equation, $T_{i}$ being the state variable and $R_{i}, Y_{i}$ control variables. The solution is similar in spirit, but the mathematics are more cumbersome. Therefore, we stick to the simpler case with constant waste to focus on the issues arising from the interaction between capacity and location.

${ }^{5}$ Note that, since $T C_{i} \equiv \phi \sum_{j=1}^{m} q_{j} d\left(P_{j}, R_{i}\right)$ is constant through time,

$$
\int_{T_{i}}^{T_{i+1}} e^{-\delta\left(t-T_{i}\right)} T C_{i} d t=\frac{T C_{i}}{\delta}\left(1-e^{-\delta \frac{Y_{i}}{Q}}\right) \quad i=0,1, \ldots K-1
$$

On the other hand, $T_{i}=\sum_{l=0}^{i-1} \frac{Y_{l}}{Q}$ is not defined for $i=0$. Therefore, the term associated to $i=0$ in (1) must be written separately from the summation.

Taking this into account, considering $T_{0}=0$, and using $\sum_{l=0}^{i-1} \frac{Y_{l}}{Q}$ instead of $T_{i}$ for $i=1, \ldots, K-1$ gives (4).

${ }^{6}$ In order to keep the analysis as simple as possible, we assume that the planner is concerned just about the costs during the planning horizon and not about the situation at the end of the horizon. One possible way to extend this analysis is to include a scrap value function evaluated at $\tau$ to summarize the future consequences (such as the amount of available land) of the the decisions made during the planning period.

${ }^{7}$ To obtain this derivative, recall

$$
\begin{aligned}
& T C_{h} \equiv \phi \sum_{j=1}^{m} q_{j} d_{j h}, \quad d_{j h} \equiv d\left(P_{j}, R_{h}\right) \equiv\left(p_{j 1}-r_{h 1}\right)^{2}+\left(p_{j 2}-r_{h 2}\right)^{2} \\
& D_{i h} \equiv d\left(R_{i}, R_{h}\right) \equiv\left(r_{i 1}-r_{h 1}\right)^{2}+\left(p_{i 2}-r_{h 2}\right)^{2}
\end{aligned}
$$


${ }^{8}$ The condition in André and Cerdá (2004, Proposition 2) is just slightly different because the construction cost function is allowed to be different from landfill to landfill $\left(C_{0}\left(Y_{0}\right), C_{1}\left(Y_{1}\right)\right.$, etc.) and hence (13) is not strictu sensu a generalization of this. Although differences in setup costs across landfills could, in principle, be incorporated here, it would complicate things without any additional insight. The reason is that, since location is endogenous, the differences in construction costs should be contingent on the location (construction cost could depend on the kind of soil, the price of land, and so on).

${ }^{9}$ Note that in Herfindahl (1967), both the location and capacity of the resource deposits are given. In André and Cerdá (2001), although different landfills may have different transportation costs, location decisions are not explicitly modelled.

\section{Appendix}

\subsection{Proof of Proposition 1}

If $K$ landfills are constructed, given the maximum capacity constraint, then $K \bar{Y} \geq \sum_{i=0}^{K-1} Y_{i}$ and using the feasibility constraint $\sum_{i=0}^{K-1} Y_{i} \geq \tau Q$, then $K \geq \frac{\tau Q}{\bar{Y}}$. If $\frac{\tau Q}{\bar{Y}}$ is an integer, then $\Psi\left(\frac{\tau Q}{\bar{Y}}\right)=\frac{\tau Q}{\bar{Y}}$. If $\frac{\tau Q}{\bar{Y}}$ is not an integer, then $K=\operatorname{Int}\left(\frac{\tau Q}{\bar{Y}}\right)$ violates the feasibility constraint and $K=\operatorname{Int}\left(\frac{\tau Q}{\bar{Y}}+1\right)$ is the smallest feasible value of $K$. Therefore we have proved the first part of the proposition. To prove the second part, suppose there is a solution given by $\left\{Y^{*}, R^{*}\right\} \equiv\left\{Y_{0}^{*}, \ldots, Y_{K-1}^{*} ; R_{0}^{*}, \ldots, R_{K-1}^{*}\right\}$ where $K>\Psi\left(\frac{\tau Q}{\underline{Y}}\right)$. Given that both $K$ and $\Psi\left(\frac{\tau Q}{\underline{Y}}\right)$ are integers by definition, then $K \geq \Psi\left(\frac{\tau Q}{\underline{Y}}\right)+1$, and given the lower bound for the capacity, the following chain of inequalities follows:

$$
\sum_{i=0}^{K-2} Y_{i} \geq(K-1) \underline{Y} \geq \Psi\left(\frac{\tau Q}{\underline{Y}}\right) \underline{Y} \geq Q \tau
$$

which implies that the combination $\left\{Y^{* \prime}, R^{* \prime}\right\} \equiv\left\{Y_{0}^{*}, \ldots, Y_{K-2}^{*} ; R_{0}^{*}, \ldots, R_{K-2}^{*}\right\}$ is a feasible solution and has a strictly smaller discounted cost than that of $\left\{Y^{*}, R^{*}\right\}$, and hence $Y^{*}$ cannot be a solution.

\subsection{Proof of Proposition 2}

Let $S_{\Theta}^{*} \equiv\left\{Y^{*}, R^{*} / \Theta\right\}$ denote the solution for problem (5) given the value of $\Theta$. If, starting from $\Theta, \tau$ or $Q$ decrease then $S_{\Theta}^{*}$ is still feasible (although not necessarily optimal), and hence $\mathcal{J}_{K}$ cannot increase. Symmetrically, when $\tau$ or $Q$ increase, $\mathcal{J}_{K}$ cannot decrease. $\phi$ and $\delta$ do not affect the feasible set, and hence, after a change in $\phi$ or $\delta, S_{\Theta}^{*}$ is still feasible. Consequently, just by computing the derivative of the objective function with respect to these parameters, it can be observed that, if $\phi$ decreases or $\delta$ increases, then $\mathcal{J}_{K}$ cannot increase and vice-versa. A decrease (increase) in $\underline{Y}$ or an increase (decrease) in $\bar{Y}$ does not directly affect the objective function, although it increases (decreases) the size of the feasible set, and hence $\mathcal{J}_{K}$ cannot decrease (increase).

\subsection{Proof of Property 1}

From condition (9), $\mu=0$. For $h=K-1$, the left-hand side of (7) is always positive. We conclude that $\alpha_{1, K-1}=0, \alpha_{0 K-1}>0$, and $Y_{K-1}=\underline{Y}$ follows from (10). 


\subsection{Example with optimal excess capacity}

Consider the following example with five cities and a linear construction cost function:

$$
\begin{aligned}
& P_{1}=(0,0), \quad q_{1}=3, \quad C(Y)=1000+10 Y, \\
& P_{2}=(1,0), \quad q_{2}=4 \text {, } \\
& P_{3}=(1,1), \quad q_{3}=2, \quad \delta=0.16, \quad \beta=0.01, \\
& P_{4}=(0,1), \quad q_{4}=1, \quad \tau=56, \quad \phi=1, \\
& P 5=(2,2), \quad q_{5}=3, \quad \underline{Y}=90, \quad \bar{Y}=400 \\
& Q=13 \text {, }
\end{aligned}
$$

The feasible region is the rectangle defined by the extreme points $(2,3)$ and $(17,16)$.

The optimal solution is given by $K^{*}=8$ landfills, and the optimal sequence of locations and capacities is:

$$
\begin{array}{llll}
R_{0}^{*}=(3.0,4.0), & Y_{0}^{*}=96, & R_{4}^{*}=(6.8,4.0), & Y_{4}^{*}=95.5, \\
R_{1}^{*}=(4.94 .0), & Y_{1}^{*}=96, & R_{5}^{*}=(2.9,7.7), & Y_{5}^{*}=90, \\
R_{2}^{*}=(3.0,5.9), & Y_{2}^{*}=96, & R_{6}^{*}=(6.7,5.8), & Y_{6}^{*}=90, \\
R_{3}^{*}=(4.9,5.9), & Y_{3}^{*}=94.5, & R_{7}^{*}=(8.7,3.9), & Y_{7}^{*}=90
\end{array}
$$

Note that

$$
\sum_{i=0}^{7} Y_{i}^{*}=748>\tau Q=728
$$

and hence there is an excess capacity equal to 20 . The discounted cost of this solution equals 3180 . The capacities $Y_{5}, Y_{6}, Y_{7}$ cannot be reduced since they are equal to the lower bound, although the capacities $Y_{0}, \ldots, Y_{4}$ could be reduced, because they are not at the lower bound. Nevertheless, if we try to improve the solution by reducing any of the capacities $Y_{0}, \ldots, Y_{4}$, then the discounted cost increases rather than decreases.

\subsection{Counter-example with nonincreasing transportation costs}

In section 3.4 we have come up with the conclusion that, for any two landfills $i<j$, with equal or very similar capacities, it must hold that $T C_{i} \leq T C_{j}$, i.e., aggregated transportation costs must be increasing. Actually, this is typically the case even if capacities are not equal. Nevertheless, when the capacities are different, it is possible to find some counter-examples such as the following. Assume the construction cost function is $C(Y)=3900+10 Y$, there are 5 cities located at the points $(0,0) ;(1,0) ;(1,1) ;(0,1)$; $(2,2)$, which produce the amounts of waste: $3,4,2,1,3$, such that $Q=13$. The feasible region is the rectangle defined by the extreme points $(2,3)$ and $(17,16)$. The rest of the parameter values are $\beta=0.01$, $\delta=0.05, \tau=56, \phi=1, \underline{Y}=90, \bar{Y}=400$. The solution is given by $K=3$ landfills, the sequence of locations $R^{*}=\{(4.9,5.9),(10.4,5.6),(3.8,10.4)\}$ and the sequence of capacities $Y^{*}=\{288,260,180\}$, as illustrated in the left-hand panel of Figure 3. The transportation costs for all the landfills turn out to be $T C_{0}=85, T C_{2}=138, T C_{3}=132$, which is not a monotonically increasing sequence. If an attempt is made to improve the solution by shifting the order of (both the capacity and location of) the second and third landfills, then total cost increases rather than decreases since the sequence of capacities is no longer optimal (Equation (13) does not hold). If the sequence of capacities is kept unchanged and the locations of the second and the third landfills are shifted, then total discounted cost decreases but the solution is 
not feasible because the security area of landfill 0 and 1 intersect, as illustrated in the right-hand panel of Figure 3 .

Acknowledge. This paper was partially written while F.J. André was visiting the department of Economics at Tilburg University. We thank the participants in the 'Sevilla Workshop on Dynamic Economics and the Environment', '1st Conference of AERNA', '13th Conference of EAERE' and '10th Conference on Computing in Economics and Finance' for their helpful comments. Comments and suggestions from two anonymous referees have improved the paper considerably. Francisco J. André is also grateful for financial support from Junta de Andalucía (project SEJ-01252), European Commission (research project EFIMAS, Proposal no. 502516) and the Spanish Ministry of Education and Science (projects SEJ2005-0508/Econ and SEJ2006-08416/ECON).

\section{References}

1. André, F.J and Cerdá, E. (2001) Optimal sequence of landfills in solid waste management. Optimal Control Applications and Methods 22: 1-25.

2. André, F.J and Cerdá, E. (2004) Landfill construction and capacity expansion. Environmental and Resource Economics 28: 409-434.

3. André, F.J and Cerdá, E. (2006) An economic analysis of municipal solid waste management and policies. In C.L. Krankhouse (ed.) Economics of Agriculture and Natural Resources. New York.

4. Beede D.N. and D.E. Bloom (1995), 'Economics of the Generation and Management of Municipal Solid Waste'. National Bureau of Economic Research, Working Paper Series No. 5116.

5. Drezner, Z. (Ed) (2002) Facility Location. Applications and Theory. Springer-Verlag, Berlin.

6. Fong, C.O. and Srinivasan, V. (1986) The multiregion dynamic capacity expansion problem: an improved heuristic. Management Science 32: 1140-1152.

7. Fullerton, D. and T. Kinnaman (eds.) (2002) The Economics of Household Garbage and Recycling behavior. New Horizons in Environmental Economics. Edward Elgar. Cheltenham, UK; Northampton, MA, USA.

8. Gaudet, G., Moreaux, M. and Salant, S.W. (1998) Intertemporal and spatial depletion of landfills, Fondazione Eni Enrico Mattei Note di Lavoro: 14/98.

9. Gaudet, G., Moreaux, M. and Salant, S.W. (2001) Intertemporal depletion of resource sites by spatially distributed users. American Economic Review 91: 1149-59.

10. Gutmann, H.M (2001) A radial basis function method for global optimization. Journal of Global Optimization 19: 201-227.

11. Hansen, P.J., Jaumard, B. and Tuy, H. (1995) Global optimization in location, in Drezner Z. (Ed) Facility Location. A Survey of Applications and Methods. Springer, New-York, 1995.

12. Hartwick, J. Kemp, M. and Long, N.V. (1986) Set-up Costs and the Theory of Exhaustible Resources. Journal of Environmental Economics and Management 13: 212-224.

13. Herfindahl, O.C. (1967) Depletion and economic theory, in M. Gaffney (Ed.), Extractive resources and taxation, pp. 63-90. Univ. of Wisconsin Press, Madison.

14. Highfill, Mc-Asey, M. and Weinstein, R. (1994) Optimality of recycling and the location of a recycling center. Journal of Regional Science 34: 583-597.

15. Holmstrom, K. (1999) The TOMLAB Optimization environment in Matlab. Advanced Modeling and Optimization 1: 47-69.

16. Horst, R. and Pardalos, P.M. (1995) Handbook of Global Optimization. Kluwer, Dordrech.

17. Huhtala, A. (1997) A post-consumer waste management model for determining optimal levels of recycling and landfilling. Environmental and Resource Economics 10: 301-314. 
18. Kunreuther, H. and Easterling, D. (1996) The role of compensation in siting hazardous facilities. Journal of Policy Analysis and Management 15: 601-622.

19. Lim, S.K. and Kim, Y.D. (1999) An integrated approach to dynamic plant location and capacity planning. Journal of the Operational Research Society 50: 1205-1216.

20. Neumaier, A. (2001) Constrained Global Optimization, in C. Bliek et al., Algorithms for solving nonlinear constrained and optimization problems: The state of the art. COCONUT Delivery D1. The Coconut Project (chapter 4). pp. 55-112. Available at http://www.mat.univie.ac.at/ neum/glopt/coconut/

21. Porter, R. (2002), The Economics of Waste. Resources for the Future. Washington, D.C.

22. Quah, E. and Tan, K. (2002) Siting environmentally unwanted facilities: Risks, trade-offs and choices. Elgar, Cheltenham, U.K. and Northampton, Mass.

23. Repa, E.W. (2000) Solid waste disposal trends. Waste Age, June.

24. Ready, M.J. and Ready, R.C. (1995) Optimal pricing of depletable, replaceable resources: the case of landfill tipping fees. Journal of Environmental Economics and Management 28: 307-323.

25. Revelle, C.S. and Eiselth, H.A. (2005) Location analysis: a synthesis and survey. European Journal of Operational Research 165: 1-19.

26. Shulman, A. (1991) An algorithm for solving dynamic capacitated plant location problems with discrete expansion sizes. Operations Research 39: 423-436.

27. Sridharan, R. (1995) The capacitated plant location problem. European Journal of Operational Research 87: 203-213.

28. Swallow, S., Opalouch, J.J. and Weaver, T.F. (1992) Siting noxious facilities: An approach that integrates technical, economic, and political considerations. Land Economics 68: 283-301.

29. US EPA (1998) The solid waste dilemma: an agenda for action. EPA-530-SW-88-054A. September. Washington.

30. Vasiloglou, V. Ch. (2004) New tool for landfill location. Waste Management and Research 22: 427-439.

\section{Figures}

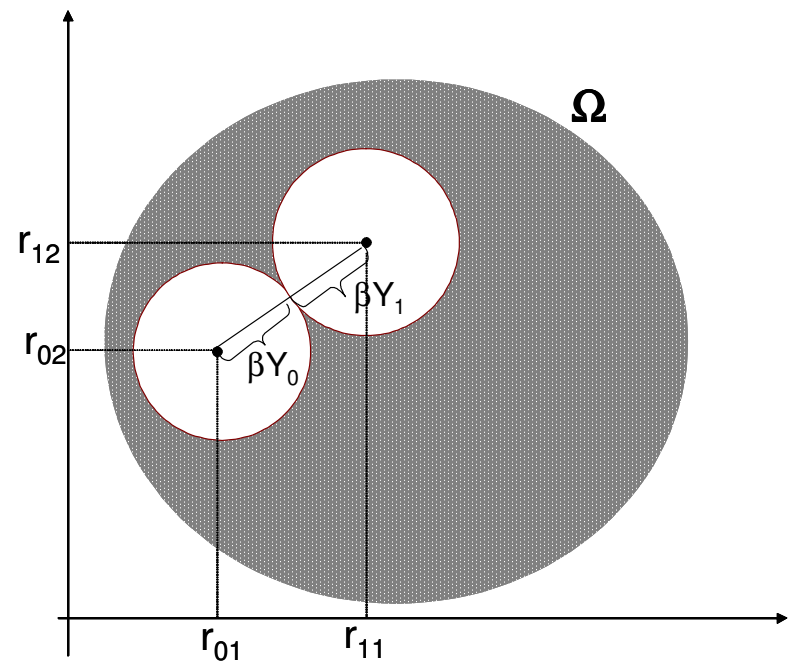

Figure 1: Interaction between capacity and location 


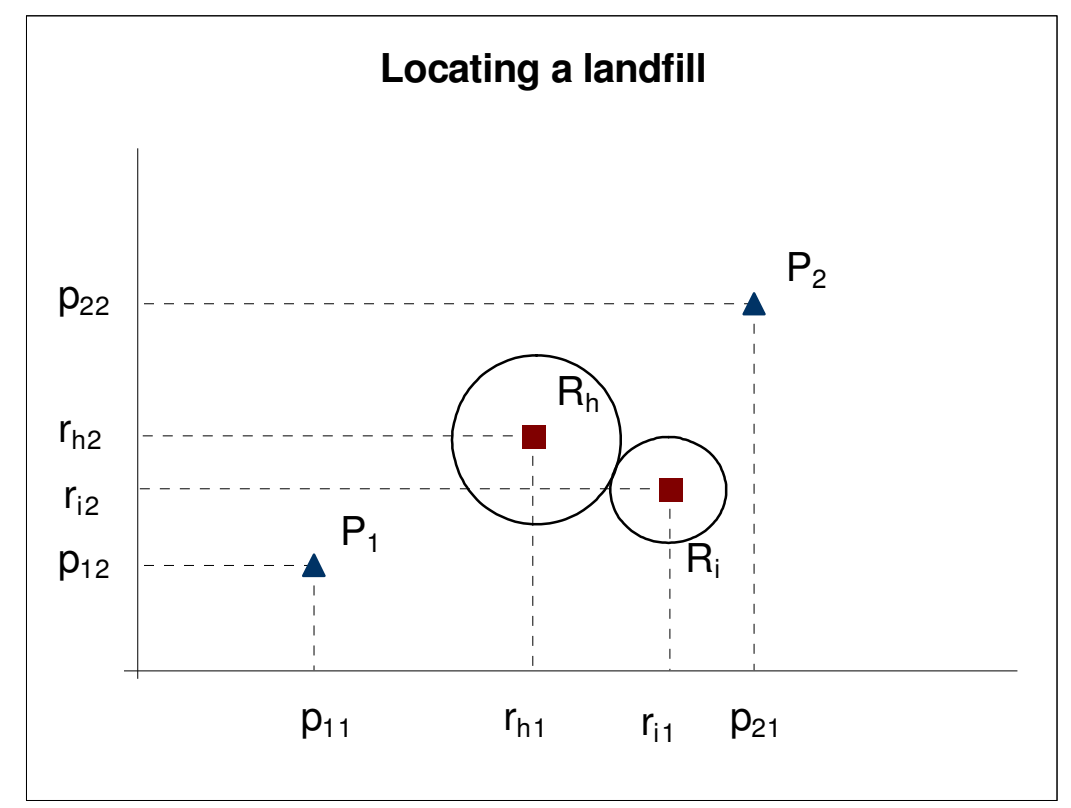

Figure 2: Determining the optimal location of a landfill
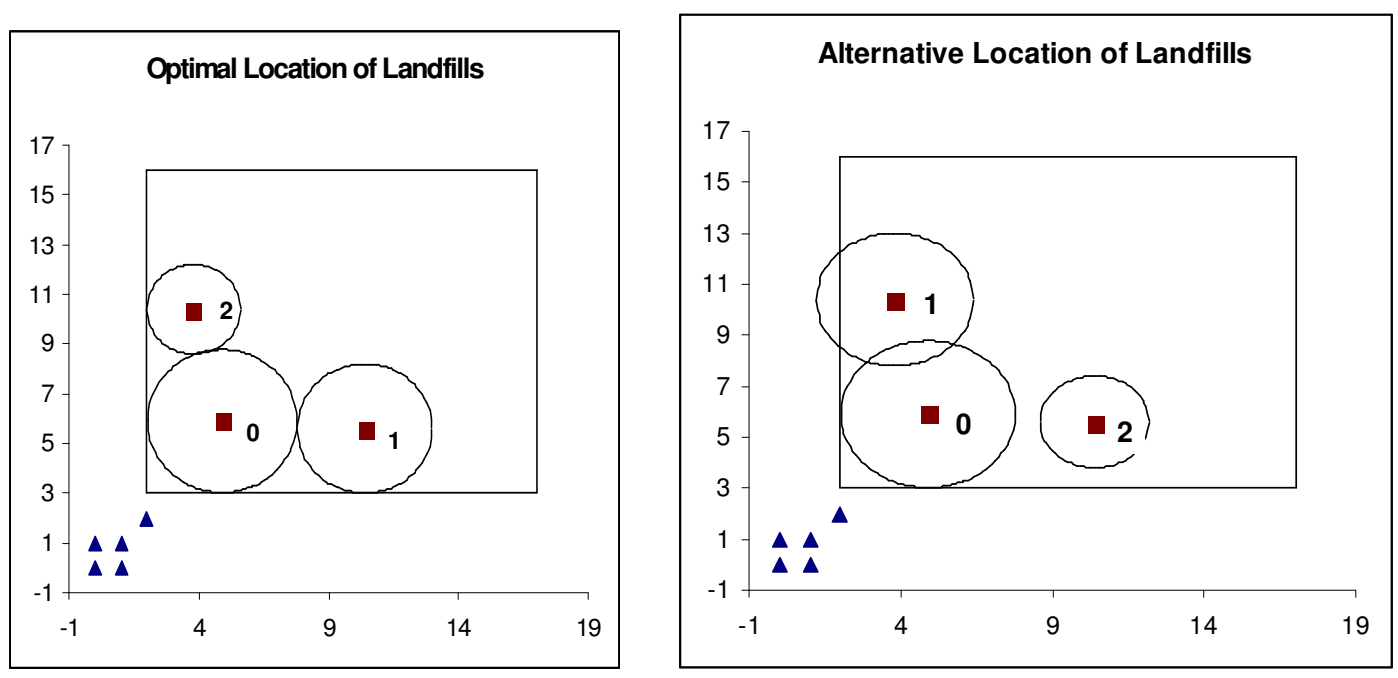

Figure 3: Example in section 3.4 

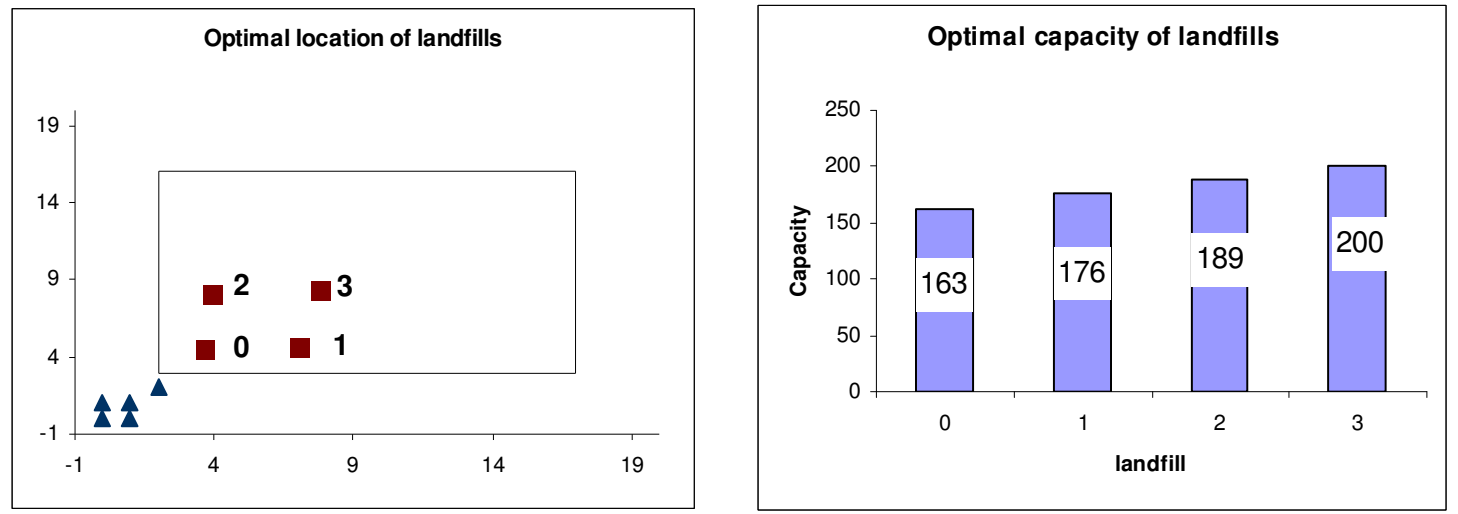

Figure 4: Optimal location and capacity of landfills in the benchmark example
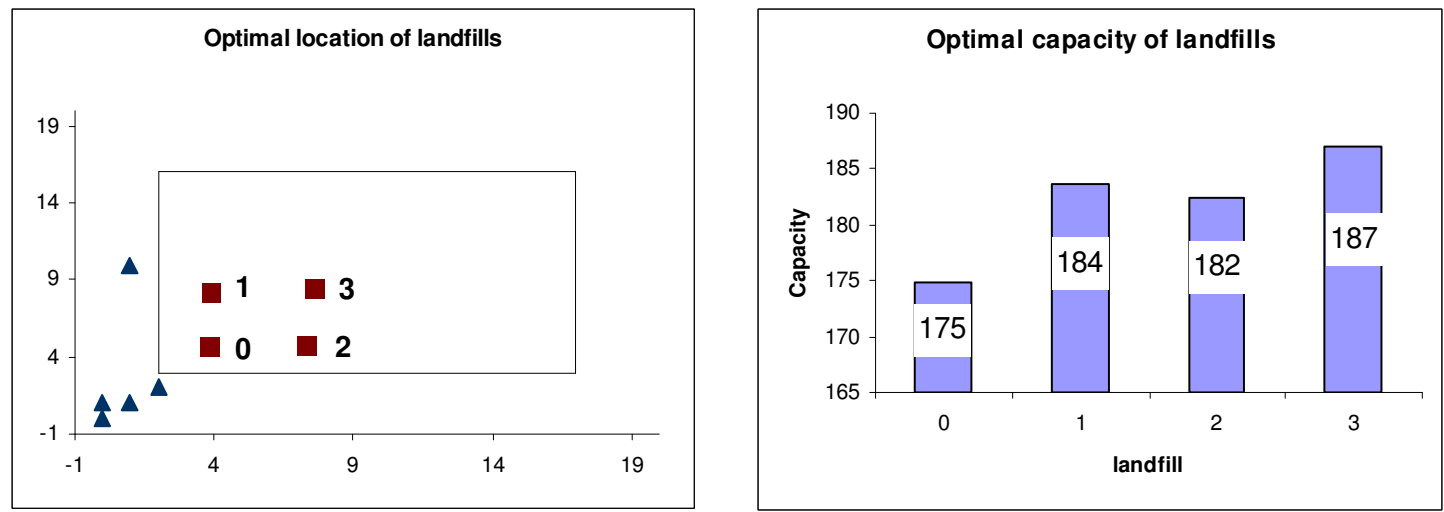

Figure 5: Optimal location and capacity after changing the location of a city
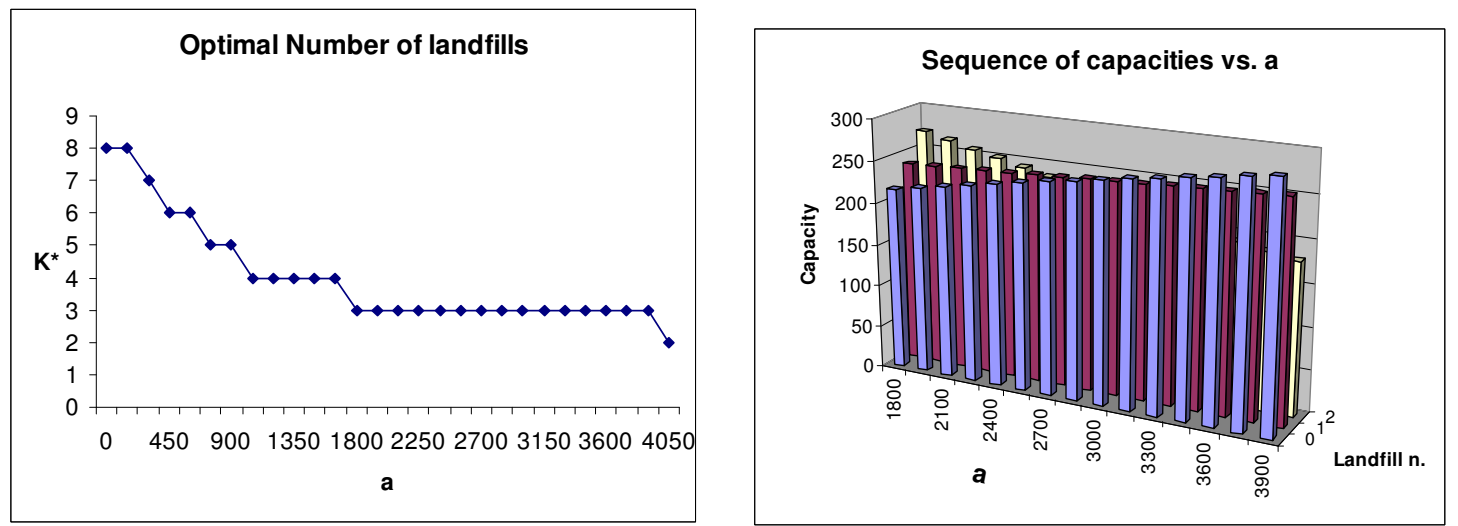

Figure 6: Impact of parameter $a$ on the solution 


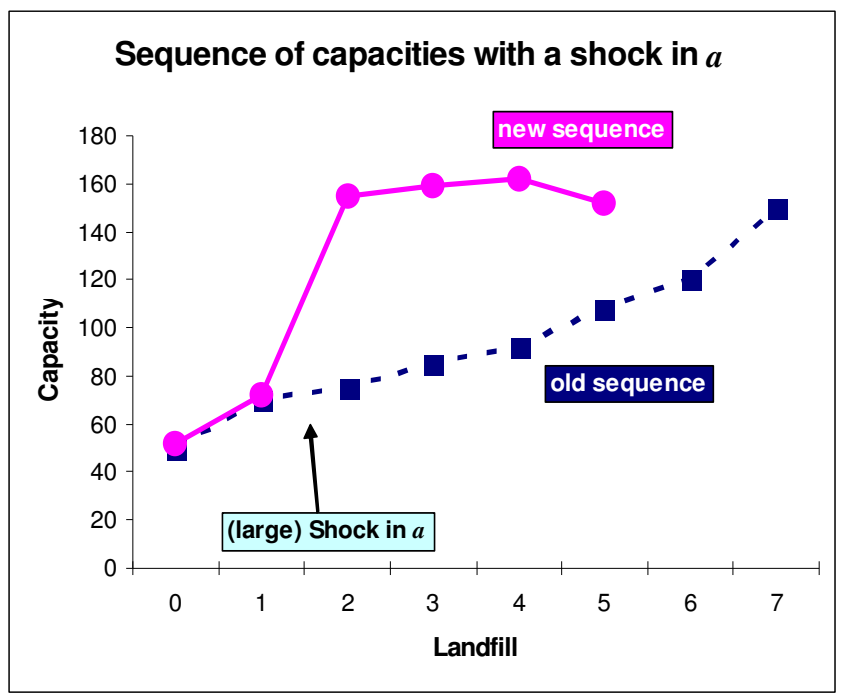

Figure 7: Determining the optimal location of a landfill
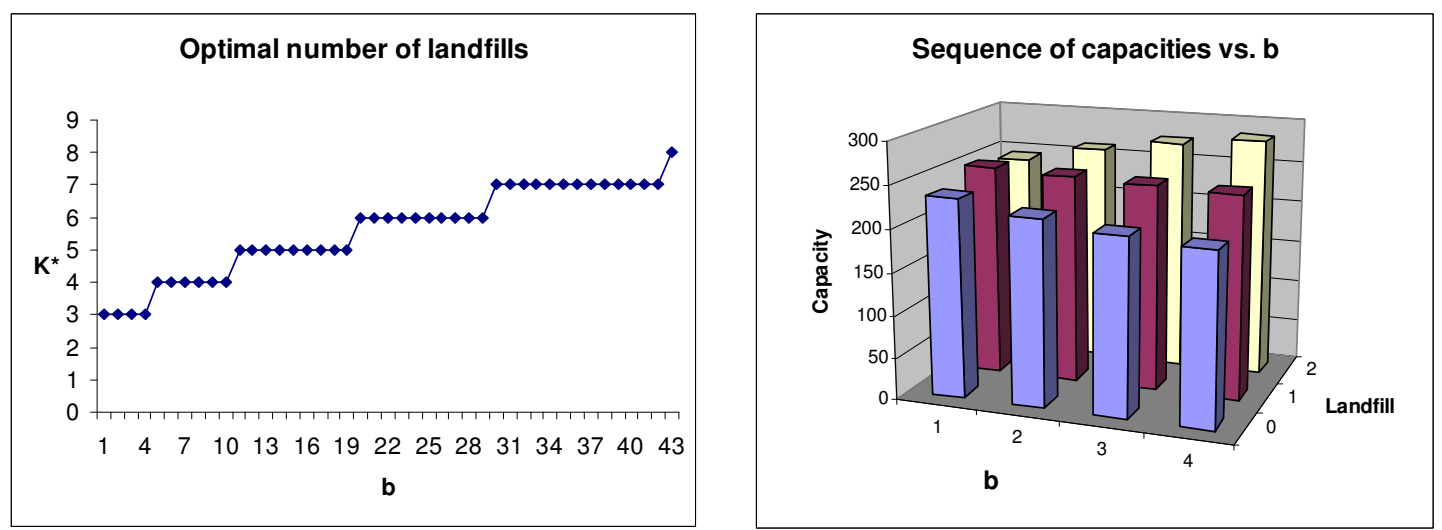

Figure 8: Impact of parameter $b$ on the solution
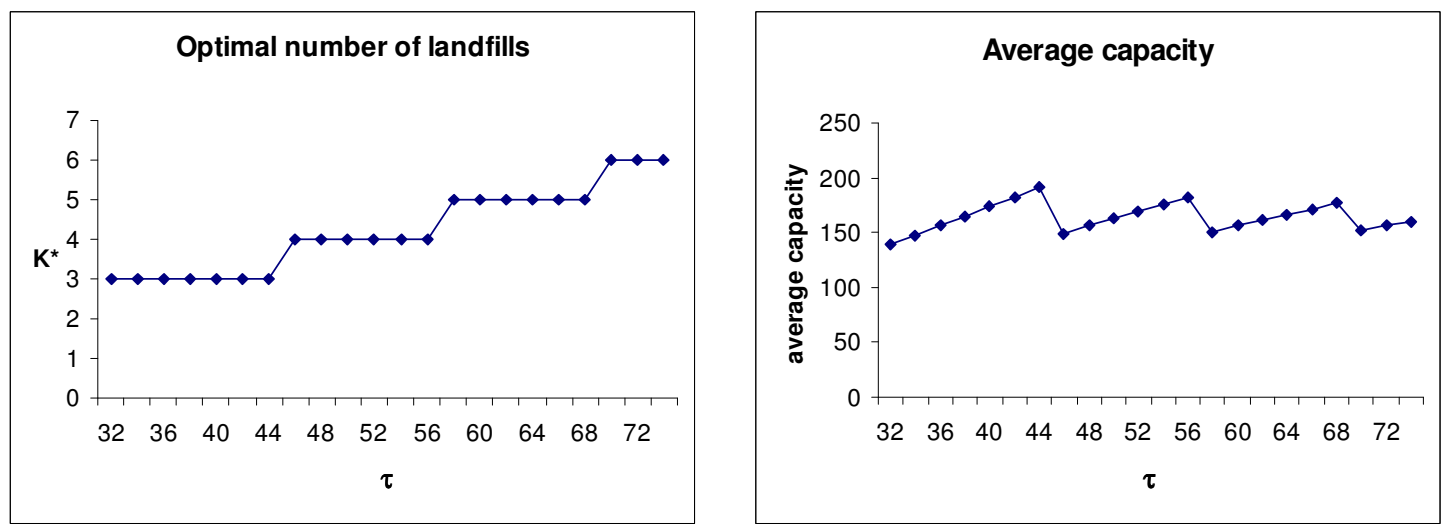

Figure 9: Impact of parameter $\tau$ on the solution 

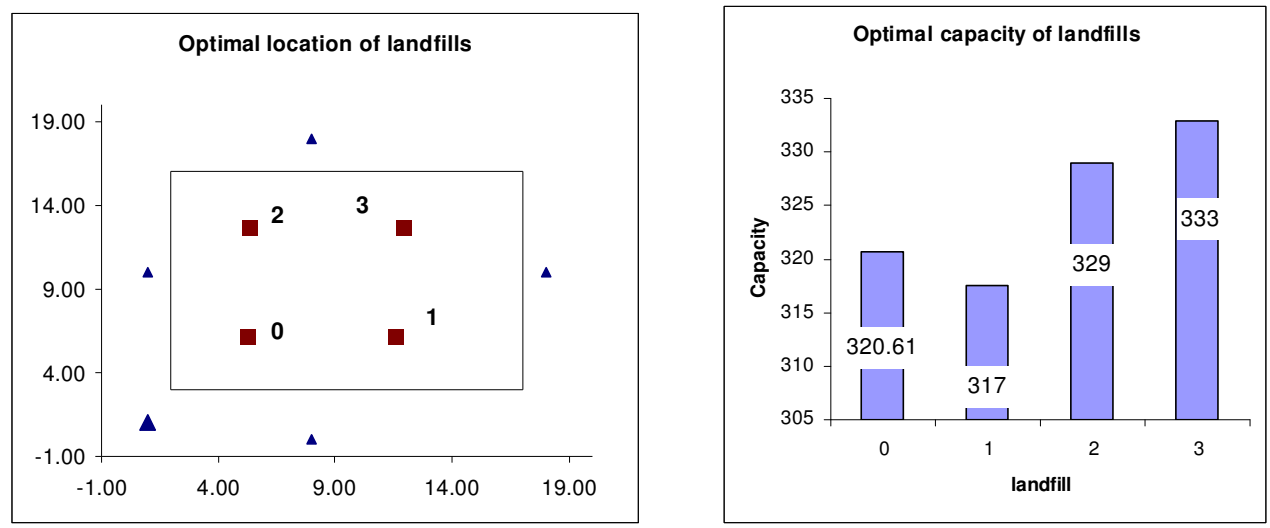

Figure 10: Solution of the example with disperse location of cities (18) 